\title{
Enhanced Thermal Conductivity for Nanofluids Containing Silver Nanowires with Different Shapes
}

\author{
Liye Zhang, ${ }^{1,2}$ Wei Yu, ${ }^{1,2}$ Dahai Zhu, ${ }^{1,2}$ Huaqing Xie, ${ }^{1,2}$ and Guiwen Huang ${ }^{3}$ \\ ${ }^{1}$ College of Engineering, Shanghai Polytechnic University, Shanghai 201209, China \\ ${ }^{2}$ Shanghai Innovation Institute for Materials, Shanghai 200444, China \\ ${ }^{3}$ Technical Institute of Physics and Chemistry, Chinese Academy of Sciences, Beijing 100190, China
}

Correspondence should be addressed to Wei Yu; yuwei@sspu.edu.cn and Guiwen Huang; gwhuang@mail.ipc.ac.cn

Received 4 August 2017; Accepted 20 September 2017; Published 10 December 2017

Academic Editor: Jingchao Zhang

Copyright (c) 2017 Liye Zhang et al. This is an open access article distributed under the Creative Commons Attribution License, which permits unrestricted use, distribution, and reproduction in any medium, provided the original work is properly cited.

\begin{abstract}
Nanofluids are the special agents to enhance the heat transfer property of the common fluids, and most of the thermal additives are the spherical nanoparticles. Up to now, the 1D thermal additives are not well exploited. In this paper, a kind of silver nanowires (AgNWs) with well-distributed shape and aspect ratio is synthesized. The results show that when we use the AgNWs prepared by the poly-vinyl-pyrrolidone (PVP) with a specific molecular weight of 40000, the thermal conductivity enhancement of nanofluids prepared by that kind of silver nanowires is as high as $13.42 \%$ when loading 0.46 vol.\% AgNWs, and the value of the thermal conductivity is $0.2843 \mathrm{~W} / \mathrm{m} \cdot \mathrm{K}$, which is far more than the case when loading the same volume of spherical silver particles. Besides, we use $\mathrm{H} \& \mathrm{C}$ model to fit the experimental results and the experimental results are consistent with the model.
\end{abstract}

\section{Introduction}

Waste heat recycling treatment has become an important issue in integrated thermal management controller [1] employed in waste heat recovery application as well as energy and refining industry [2], including petroleum, metallurgy, and mineral. Actually, the cooling technology tends to apply in smaller-shaped and faster-operated microelectronic components, higher power generators, and brighter optics due to their increasing thermal load [3]. The principle of cooling technology is to enhance heat dissipation, and the coolant with excellent thermal physic characteristics is in strong need. As a result, some new kinds of suspensions with strong heat transfer performance appear; they are the so-called "nanofluids," which disperse metal or nonmetallic nanopowder into the base fluids, such as water, ethylene glycol, oil, or other fluids.

The thermal conductivity of the base liquid is very low, so adding metal or nonmetallic nanopowder is a viable way to enhance the base fluid's thermal conductivity. Previously, the additives are commonly the micron-sized particles, but the properties are always affected by the particle size, morphology, particle agglomeration, settlement, interface thermal resistance, and so on [4]. Considering the above-mentioned issues, there is an urgent need to develop a kind of nanofluid avoiding the disadvantages to the utmost extent. Through more than two decades' development, many nanomaterials are tried to be as the thermal conductive additives, containing metal, metal oxide, and carbon-based materials. Fedele et al. reported $20 \sim 33 \%$ enhancement when the $\mathrm{TiO}_{2}$ fraction was varying from $1 \%$ to $35 \%$ [5]. With the $0.001 \% \sim 1$ vol. $\% \mathrm{CuO}$ nanoparticles, the maximum of composites' thermal conductivity could run up to $4.8 \%$, as reported by Kwak and Kim [6].

Among the thermal conductive additives, the carbonbased materials (carbon nanotubes and graphene) attract much attention. Speaking of carbon nanotubes, despite having high aspect ratio, they are hydrophobic so that they will form beams through the interaction between $\pi-\pi$ bonds. However, it is hard for these beams to disperse well, which in turn will weaken the chemical stability and thermal performance of the nanofluids [7]. Graphene is just like carbon nanotubes, and its dispersion is also a large problem. Graphene oxide with rich functional group is a good choice, but it is not stable; heat or light can reduce graphene oxide to graphene. Yu et al. $[8,9]$ reported that they prepared a kind of stable ethylene-glycol-based nanofluid containing graphene 


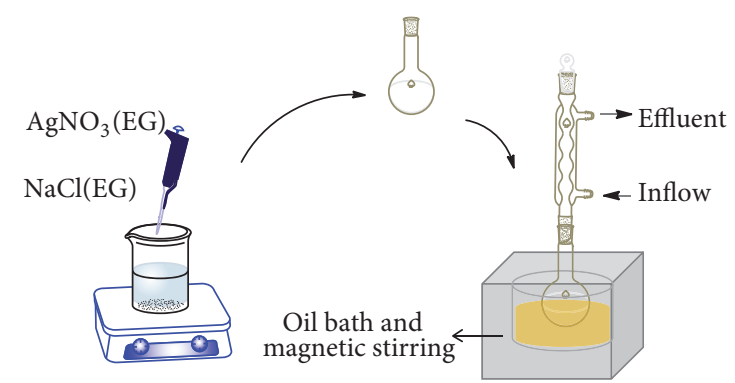

FIGURE 1: Schematic diagram of the preparation process.

oxide nanosheets. When loading a little volume of graphene oxide nanosheets, thermal conductivity will increase rapidly to a high degree. This kind of nanofluids can keep stable thermal conductivity for 7 days, which indicates its strong stability.

Metal nanofillers with outstanding electrical properties and thermal conductivity are widely used in composites. The shape of nanofillers has great influence on the properties of composites, especially for the 1D metal fillers with high aspect ratio. Zeng's group found that silver nanowires (AgNWs) network had fantastic optoelectronic performance compared to indium tin oxide (ITO) [10]. Because of the relatively low transmittance and poor electrical conductivity of carbonbased nanomaterials and the high cost of indium source and inflexibility of ITO [11], AgNWs have become a prerequisite alternative to make transparent conductive electrodes. In this paper, we prepared AgNWs with different shapes and tried to explore the potential of enhancing the nanofluid's thermal conductivity with AgNWs.

\section{Materials and Methods}

The silver nitrate $\left(\mathrm{AgNO}_{3}, 99.8 \%\right)$, sodium chloride $(\mathrm{NaCl}$, 99.8\%), and ethylene glycol (EG, 99\%) used to obtain our product were all purchased from Sinopharm Group Chemical Reagent Co., Ltd. $\mathrm{PVP}_{\mathrm{MW}=1300000}$ and $\mathrm{PVP}_{\mathrm{MW}=40000}$ were purchased from Aladdin (Shanghai, People's Republic of China).

We used a convenient and fast approach for synthesis of silver nanowires based on the work published by Song et al. [12], and the preparation process is shown in Figure 1. In a synthesis process, $1 \mathrm{ml} \mathrm{AgNO}_{3}$ solution (0.9 M in EG) and $0.6 \mathrm{ml} \mathrm{NaCl}$ solution (0.01 M in EG) were quickly put into $18.4 \mathrm{ml}$ PVP solution (0.286 $\mathrm{M}$ in EG). Then the mixture was moved into a round-bottomed flask under oil bath magnetic stirring at the temperature of $185^{\circ} \mathrm{C}$ for 20 minutes without warming up from room temperature. After this process, we acquired the final product by centrifuging it 3 times with deionized water at a rotating speed of $10000 \mathrm{rpm} / \mathrm{min}$ to remove the extra PVP and EG. Centrifugation can keep all the products as much as possible so that we can do better analysis.

Through the above method, we can achieve the pure AgNWs. Then, it is dispersed in EG and treated for 2 hours under ultrasonication to obtain stable AgNWs/EG nanofluids.

We observed the morphologies of AgNWs by scanning electron microscopy (SEM, Hitachi S4800, Japan) and transmission electron microscopy (TEM, 2100F, JEOL, Japan). And the thermal conductivity of nanofluids with AgNWs was tested by $\mathrm{Tci}^{\mathrm{TM}}$ (C-Therm Technologies Ltd., Canada). This kind of test instrument uses the patent technology of Modified Transient Plane Source (MTPS). The whole system composes a heat sensor, a power control device, and data collection software. On the top of the sensor there is a cylindrical place where the test sample is placed with about $2 \mathrm{~mm}$ height. When the whirlpool heating source at the center of the sensor generates heat which passes the sensor into the material we are testing, the voltage at the heating source shows a sharp decrease. As a result, we obtain the thermal conductivity through the data of voltage decrease. Every sample is tested for 5 times in order to acquire a reliable average value, under a controlled $25^{\circ} \mathrm{C}$ and within $0 \sim 100 \mathrm{~W} / \mathrm{m} \cdot \mathrm{K}$ and $-50 \sim 200^{\circ} \mathrm{C}$ range. The accuracy is controlled within $\pm 1 \%$.

\section{Results and Discussion}

In this manuscript, we try to investigate how the molecular weight (MW) of PVP affects the morphology of AgNWs and how the shape of AgNWs further affects the thermal performance of AgNWs contained nanofluids. During the synthesis progress of AgNWs, silver nitrate acts as the precursor, ethylene glycol acts as the reducing agent, PVP acts as the capping agent, and it can control the aspect ratio of AgNWs.

We can see the different morphologies of AgNWs when adding PVP with different MW as Figures 2(a) and 2(b) show. When the MW is 40000 , the products are almost linear AgNWs. When the MW is 1300000 , the products are the mixture of AgNWs and spherical particles, and most of them are spherical. This strongly suggests that the MW has a huge effect on morphology of Ag nanoparticles. The length distribution and aspect ratio distribution of $\mathrm{PVP}_{\mathrm{MW}=40000}$ (K30) are calculated as shown in Figures 3(a) and 3(b). We find that the length distribution as well as aspect ratio distribution of K30 are basically consistent with the normal distribution. It illustrates the uniformity of the nanowires. The length of nanowires is around $1 \mathrm{um}$, and the aspect ratio of nanowires is about 25 .

As Figure 4 shows, the FTIR spectra of AgNWs prepared by PVP of different MW have some consistent peaks. The peaks around $1635 \mathrm{~cm}^{-1}$ are assigned to the stretching vibration of $-\mathrm{C}=\mathrm{O}$, which indicates the possible function of carbonyl group. The absorption peaks at $3428 \mathrm{~cm}^{-1}$ are due to $-\mathrm{OH}$ (hydroxyl groups) stretching vibration. Compared with pure PVP, the carbonyl absorption peak of AgNWs in nanofluids shifts from 1659 to $1635 \mathrm{~cm}^{-1}$; this may be due to carbonyl groups involving in the reaction, and the oxygen atom in the carbonyl group incorporates part of the AgNWs [13]. Consequently, we can conclude that there is some interaction between PVP and silver, and the MW of PVP is the important influence factor to control the morphologies of Ag nanoparticles.

As is shown in Figure 5(a), it is a single Ag nanowire, and it is linear and smooth. Most AgNWs are linear, not like CNTs. The aspect ratio of the single AgNW is measured initially as 24.6 , which corresponds to the statistical results 


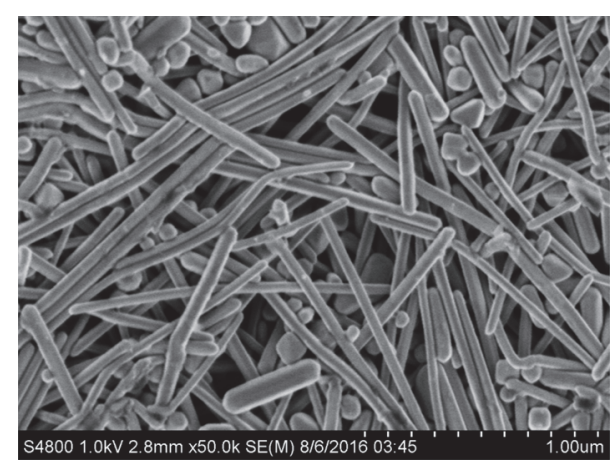

(a)

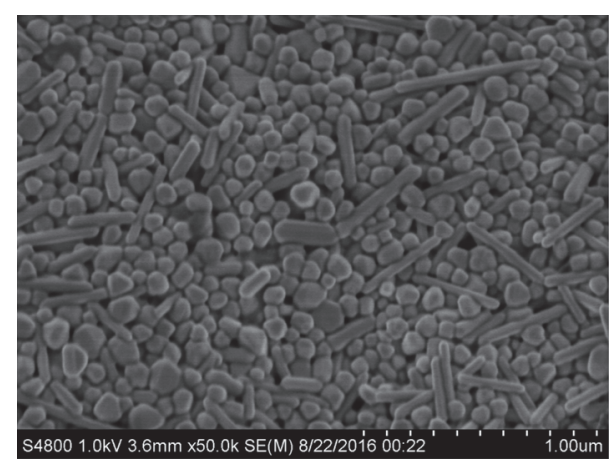

(b)

Figure 2: SEM images of AgNWs when adding (a) $\mathrm{PVP}_{\mathrm{MW}=40000}$ and (b) $\mathrm{PVP}_{\mathrm{MW}=130000}$.

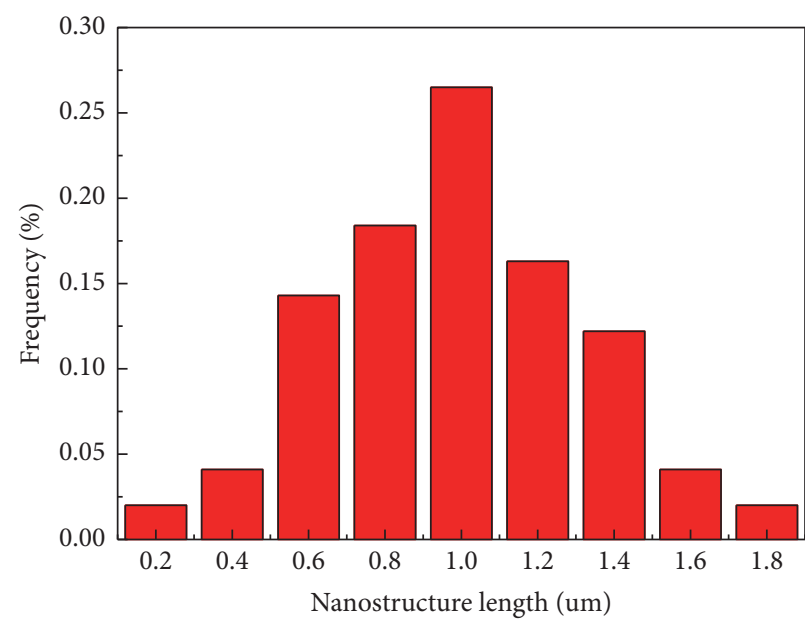

(a)

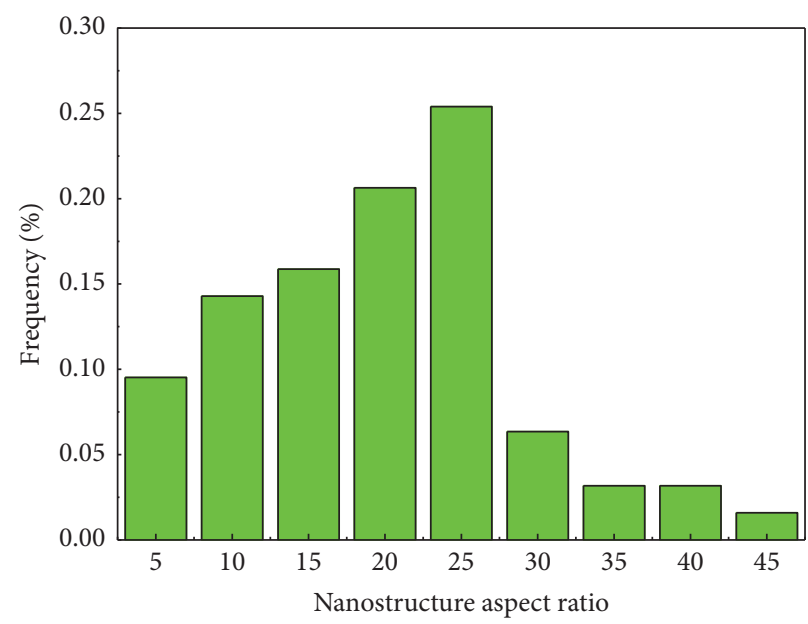

(b)

FIgURE 3: The length distributions (a) and aspect ratio distribution (b) of AgNWs with $\mathrm{PVP}_{\mathrm{MW}=40000}$.

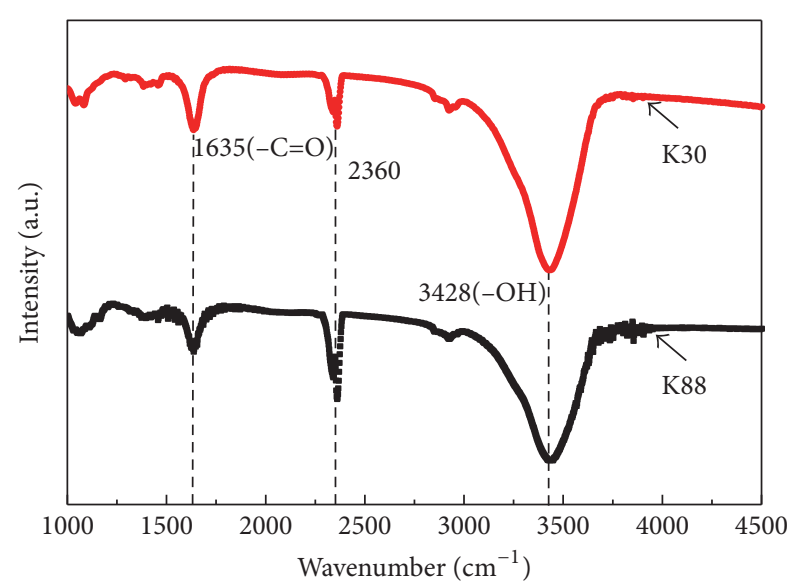

FIGURE 4: FTIR spectra of AgNWs synthesized by PVP with different MW.

above. Figure 5(b) shows the selected area electron diffraction (SAED) pattern of AgNWs. The high-resolution TEM (HRTEM) of the edge of this AgNWs is shown in Figure 5(c).
The crystal distance we calculated from the corresponding SAED pattern is $0.232 \mathrm{~nm}$, which is consistent with its $\{111\}$ plane.

In order to investigate heat transfer performance of AgNWs, we prepared ethylene-glycol-based nanofluids containing certain percentage of AgNWs. As seen from Figure 6, two kinds of these nanofluids' thermal conductivity were measured. With the increase of volume fraction of AgNWs, the thermal conductivity of both nanofluids is improving at a certain upward trend. The thermal conductivity enhancements which represent the improved thermal conductivity relative to that of the base fluid EG for AgNWs and nanoparticles contained nanofluids are $13.72 \%$ and $4.76 \%$, respectively, when the volume fraction of AgNWs is 0.46 vol. $\%$. The reason for this result may be that the aspect ratio of AgNWs (K30) is far greater than $\mathrm{Ag}$ nanoparticles (K88) and that greatly enhances the heat transfer efficiency [14-16]. It is pointed that the trend of thermal conductivity enhancement of nanofluids is almost like a straight line.

There have been a lot of models about nanofluids' thermal conductivity. The models established by Maxwell [17] and Hamilton and Crosser [18] are compliant to cases when 


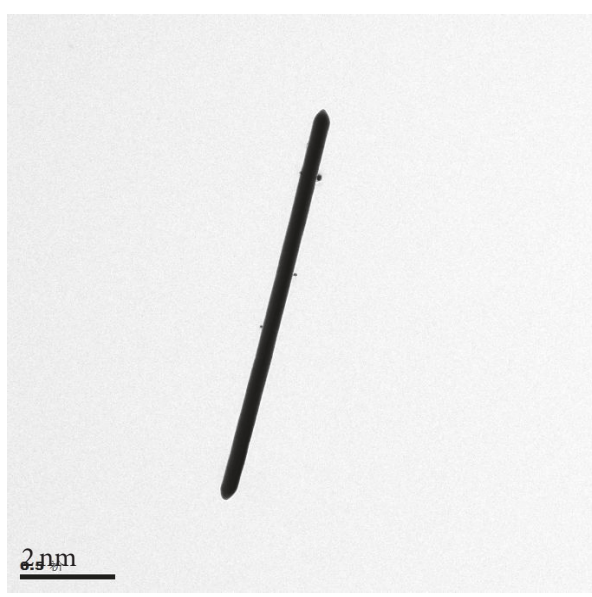

(a)

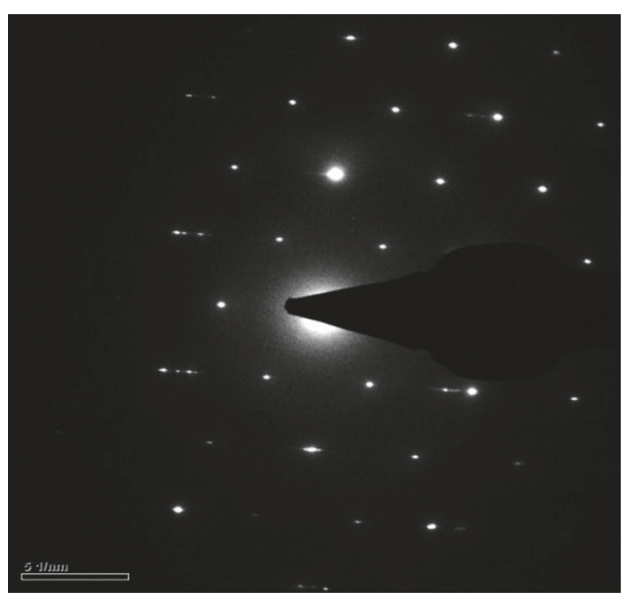

(b)

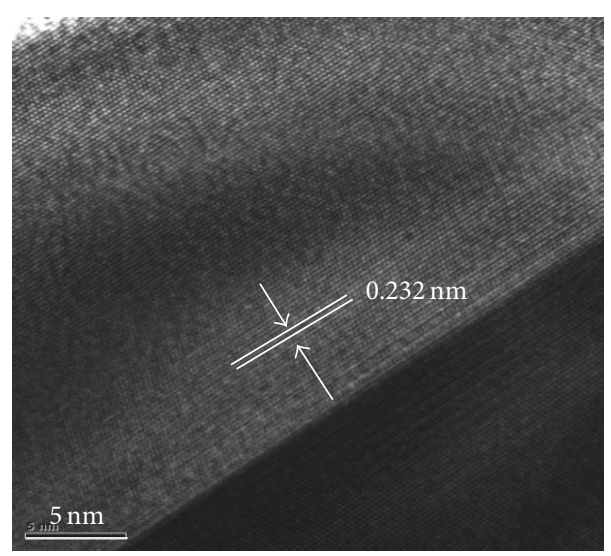

(c)

Figure 5: $(\mathrm{a}-\mathrm{c}) \mathrm{TEM}$ characterizations of AgNWs synthesized in $\mathrm{PVP}_{\mathrm{MW}=40000}$.

nonspherical particles act as granular discontinuous phase dissolved in materials, and both of them consider the effect of particle shapes on the overall thermal conductivity model. In this paper, the Hamilton and Crosser model ( $\mathrm{H} \& \mathrm{C}$ model) was adopted to predict the thermal conductivity. The equation is presented as

$$
\frac{K_{c}}{K_{e}}=\frac{K_{a}+(n-1) K_{e}-(n-1) V_{a}\left(K_{e}-K_{a}\right)}{K_{a}+(n-1) K_{e}+V_{a}\left(K_{e}-K_{a}\right)} .
$$

In this equation, the parameters $K_{c}, K_{a}$, and $K_{e}$ represent the thermal conductivity of the nanofluids containing AgNWs, the discontinuous phase AgNWs, and the continuous phase ethylene glycol, respectively. $V_{a}$ is the volume fraction of discontinuous phase. $X=n-1$ is called shape factor, which indicates the dependence of particle shape on the varieties of thermal conductivity. Actually, $n$ is a function of $K_{a}$ and $K_{e}$ [19], and $n=3 / \psi$, where $\psi$ is the sphericity, which means the ratio of the surface area of the sphere of the same volume to the surface of the object and the surface area of the object. When the aspect ratio of AgNWs is different, then $X$ will vary correspondingly following the rule

$$
X=2 \psi^{0.2}\left(\frac{l_{p}}{l_{d}}\right),
$$

where $l_{p} / l_{d}$ represents the aspect ratio of AgNWs. The fitting curves of the thermal conductivity of AgNWs nanofluids prepared by two kinds of PVP (K30 and K88) are shown in Figure 7. We can see that the predictive values based on $\mathrm{H} \& \mathrm{C}$ model of thermal conductivity follow a stable straight line trend of increasing as the volume fraction of AgNWs addition increases in nanofluids. Meanwhile, the actual values of thermal conductivity are floating around the fitting curve with a very small range. Taking the model of nanofluids prepared with PVP (K30) as an example, from the law of change of thermal conductivity in Figure 7, we can calculate that the value of $X$ is 28 through (1), and then we can obtain the value of $\psi$ via the relationship " $n=3 / \psi$ "; substituting $\psi$ into (2), we finally obtain the aspect ratio of AgNWs synthesized by PVP (K30) around 22 that is similar to the previous statistics shown in Figure 3, which indicates that the prediction of the $\mathrm{H} \& \mathrm{C}$ model is such a match to the actual values of thermal conductivity. Hence, we can consider 


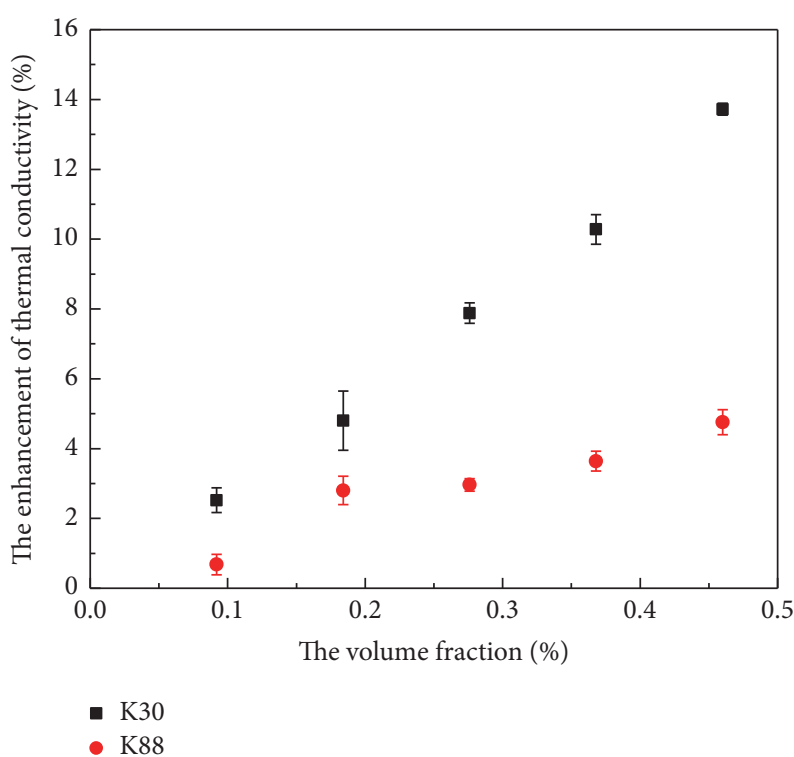

Figure 6: Thermal conductivity enhancement of AgNWs nanofluids synthesized by $\mathrm{PVP}_{\mathrm{MW}=40000}$ and $\mathrm{PVP}_{\mathrm{MW}=1300000}$.

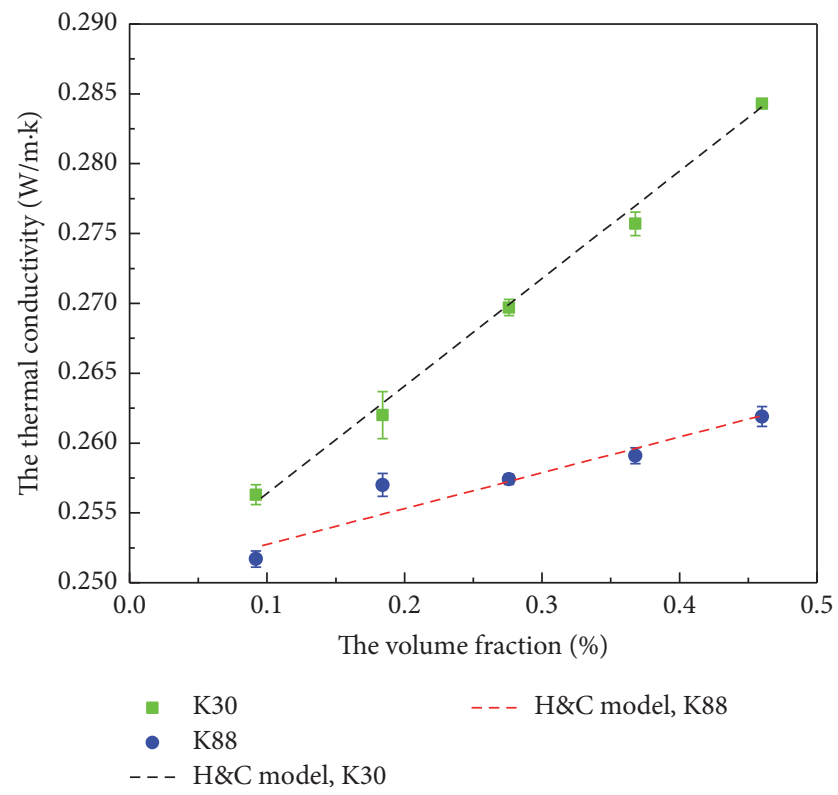

Figure 7: H\&C model fitting of AgNWs nanofluids prepared by $\mathrm{PVP}_{\mathrm{MW}=40000}$ and $\mathrm{PVP}_{\mathrm{MW}=1300000}$.

applying $\mathrm{H} \& \mathrm{C}$ model to predict thermal conductivity in kindred nanowires-based nanofluids.

\section{Conclusion}

In conclusion, the molecular weight of PVP has the great influence on the shape of silver nanowires and the thermal conductivity of silver nanowires nanofluids. Under the volume fraction of $0.46 \mathrm{vol} . \%$, the thermal conductivity of nanofluids containing silver nanowires is $0.2843 \mathrm{~W} / \mathrm{m} \cdot \mathrm{K}$, far greater than that containing spherical silver particles, whose thermal conductivity is $0.2619 \mathrm{~W} / \mathrm{m} \cdot \mathrm{K}$. As a result, we can conclude that nanowires are the trend of nanofluids additive in the future, which can significantly enhance the thermal conductivity of nanofluids. When we use the H\&C model to fit the experimental data, and they are in a good agreement, it indicates that we can use the H\&C model to predict the thermal conductivity value.

\section{Conflicts of Interest}

The authors declare that there are no conflicts of interest regarding the publication of this paper.

\section{Acknowledgments}

The work was supported by National Natural Science Foundation of China (51476094 and 51590902), Shu Guang project supported by Shanghai Municipal Education Commission and Shanghai Education Development Foundation (15SG52), and the Key Subject of Shanghai Polytechnic University (Material Science and Engineering, XXKZD1601).

\section{References}

[1] S. Li, J. Y. Wu, R. Z. Wang, and Y. Huangfu, "Study of heat and mass transfer in integrated thermal management controller (ITMC) employed in waste heat recovery application," Energy Conversion and Management, vol. 48, no. 12, pp. 3074-3083, 2007.

[2] N. Maruoka, T. Mizuochi, H. Purwanto, and T. Akiyama, "Feasibility Study for Recovering Waste Heat in the Steelmaking Industry Using a Chemical Recuperator," ISIJ International, vol. 44, no. 2, pp. 257-262, 2004.

[3] P. Keblinski, J. A. Eastman, and D. G. Cahill, "Nanofluids for thermal transport," Materials Today, vol. 8, no. 6, pp. 36-44, 2005.

[4] W. Evans, R. Prasher, J. Fish, P. Meakin, P. Phelan, and P. Keblinski, "Effect of aggregation and interfacial thermal resistance on thermal conductivity of nanocomposites and colloidal nanofluids," International Journal of Heat and Mass Transfer, vol. 51, no. 5-6, pp. 1431-1438, 2008.

[5] L. Fedele, L. Colla, and S. Bobbo, "Viscosity and thermal conductivity measurements of water-based nanofluids containing titanium oxide nanoparticles," International Journal of Refrigeration, vol. 35, no. 5, pp. 1359-1366, 2012.

[6] K. Kwak and C. Kim, "Viscosity and thermal conductivity of copper oxide nanofluid dispersed in ethylene glycol," KoreaAustralia Rheology Journal, vol. 17, no. 2, pp. 35-40, 2005.

[7] H. Kuzmany, J. Fink, M. Mehring, and S. Roth, "Molecular Nanostructures," in Proceedings of the International Winterschool on Electronic Properties of Novel Materials, pp. 1-570, Kirchberg/Tyrol, Austria.

[8] W. Yu, H. Xie, and D. Bao, "Enhanced thermal conductivities of nanofluids containing graphene oxide nanosheets," Nanotechnology, vol. 21, no. 5, Article ID 055705, 2010.

[9] W. Yu, H. Xie, X. Wang, and X. Wang, "Significant thermal conductivity enhancement for nanofluids containing graphene nanosheets," Physics Letters A, vol. 375, no. 10, pp. 1323-1328, 2011.

[10] X.-Y. Zeng, Q.-K. Zhang, R.-M. Yu, and C.-Z. Lu, "A new transparent conductor: Silver nanowire film buried at the surface of 
a transparent polymer," Advanced Materials, vol. 22, no. 40, pp. 4484-4488, 2010.

[11] A. R. Madaria, A. Kumar, and C. Zhou, "Large scale, highly conductive and patterned transparent films of silver nanowires on arbitrary substrates and their application in touch screens," Nanotechnology, vol. 22, no. 24, Article ID 245201, 2011.

[12] J. Y. Song, M. Wang, Y. X. Zhang et al., "Investigation on the role of the molecular weight of polyvinyl pyrrolidone in the shape control of high-yield silver nanospheres and nanowires," Nanoscale Research Letters, vol. 9, no. 1, p. 17, 2014.

[13] X. Yang and Y. Lu, "Preparation of polypyrrole-coated silver nanoparticles by one-step UV-induced polymerization," Materials Letters, vol. 59, no. 19-20, pp. 2484-2487, 2005.

[14] F. Deng, Q.-S. Zheng, L.-F. Wang, and C.-W. Nan, "Effects of anisotropy, aspect ratio, and nonstraightness of carbon nanotubes on thermal conductivity of carbon nanotube composites," Applied Physics Letters, vol. 90, no. 2, Article ID 021914, 2007.

[15] J. W. Shan, "Particle aspect-ratio effects on the thermal conductivity of micro-and nanoparticle suspensions," Journal of Heat Transfer, vol. 130, no. 8, pp. 318-323, 2008.

[16] R. S. Kapadia, B. M. Louie, and P. R. Bandaru, "The influence of carbon nanotube aspect ratio on thermal conductivity enhancement in nanotube-polymer composites," Journal of Heat Transfer, vol. 136, no. 1, Article ID 011303, 2014.

[17] J. C. Maxwell, "A Treatise on Electricity and Magnetism 2," Nature, vol. 7, no. 182, pp. 478-480, 1904.

[18] L. R. Hamilton and O. K. Crosser, "Thermal conductivity of heterogeneous two-component systems," Industrial \& Engineering Chemistry Fundamentals, vol. 1, no. 3, pp. 27-40, 1962.

[19] H. Fricke, "A mathematical treatment of the electric conductivity and capacity of disperse systems I. The electric conductivity of a suspension of homogeneous spheroids," Physical Review A: Atomic, Molecular and Optical Physics, vol. 24, no. 5, pp. 575$587,1924$. 

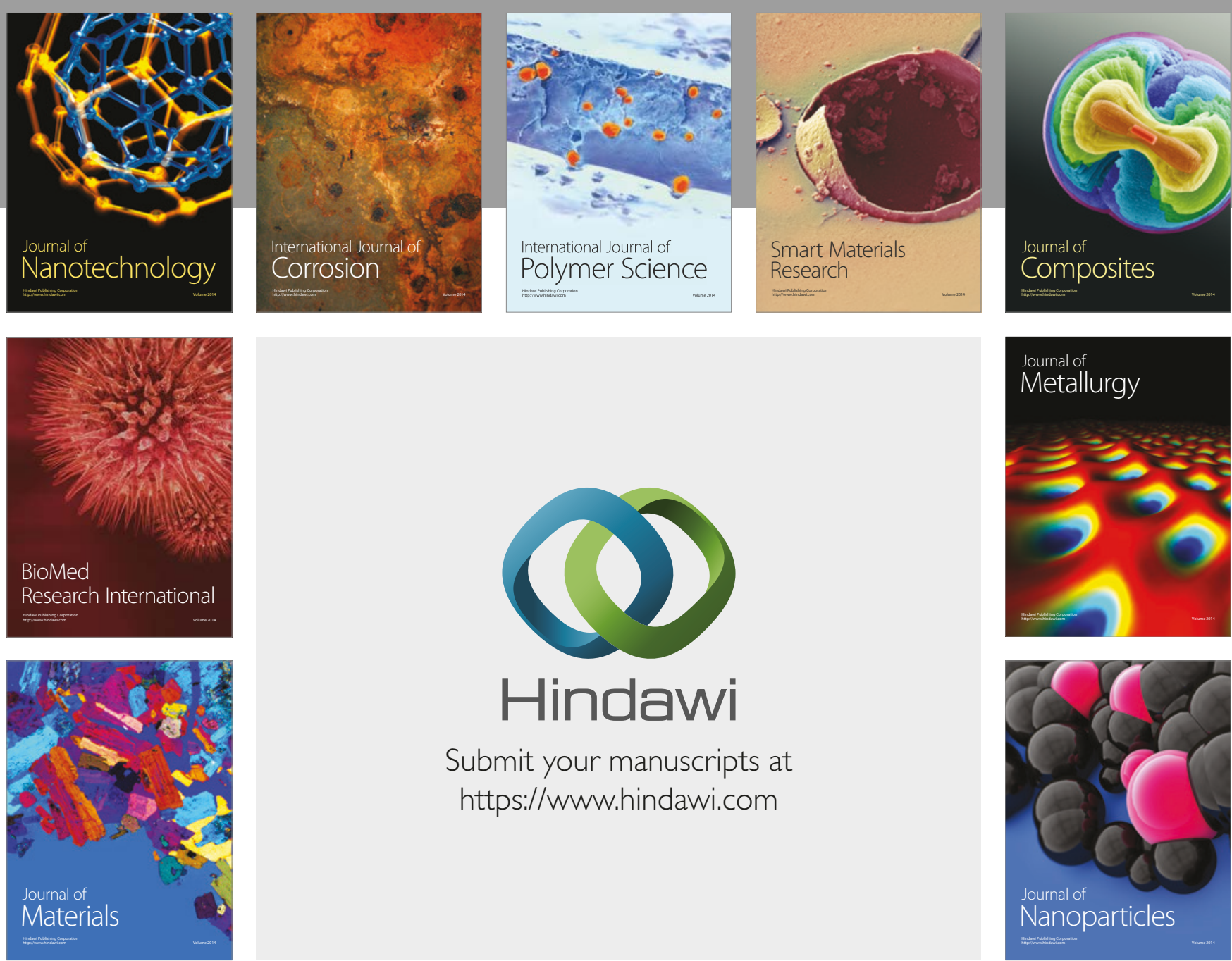

\section{Hindawi}

Submit your manuscripts at

https://www.hindawi.com
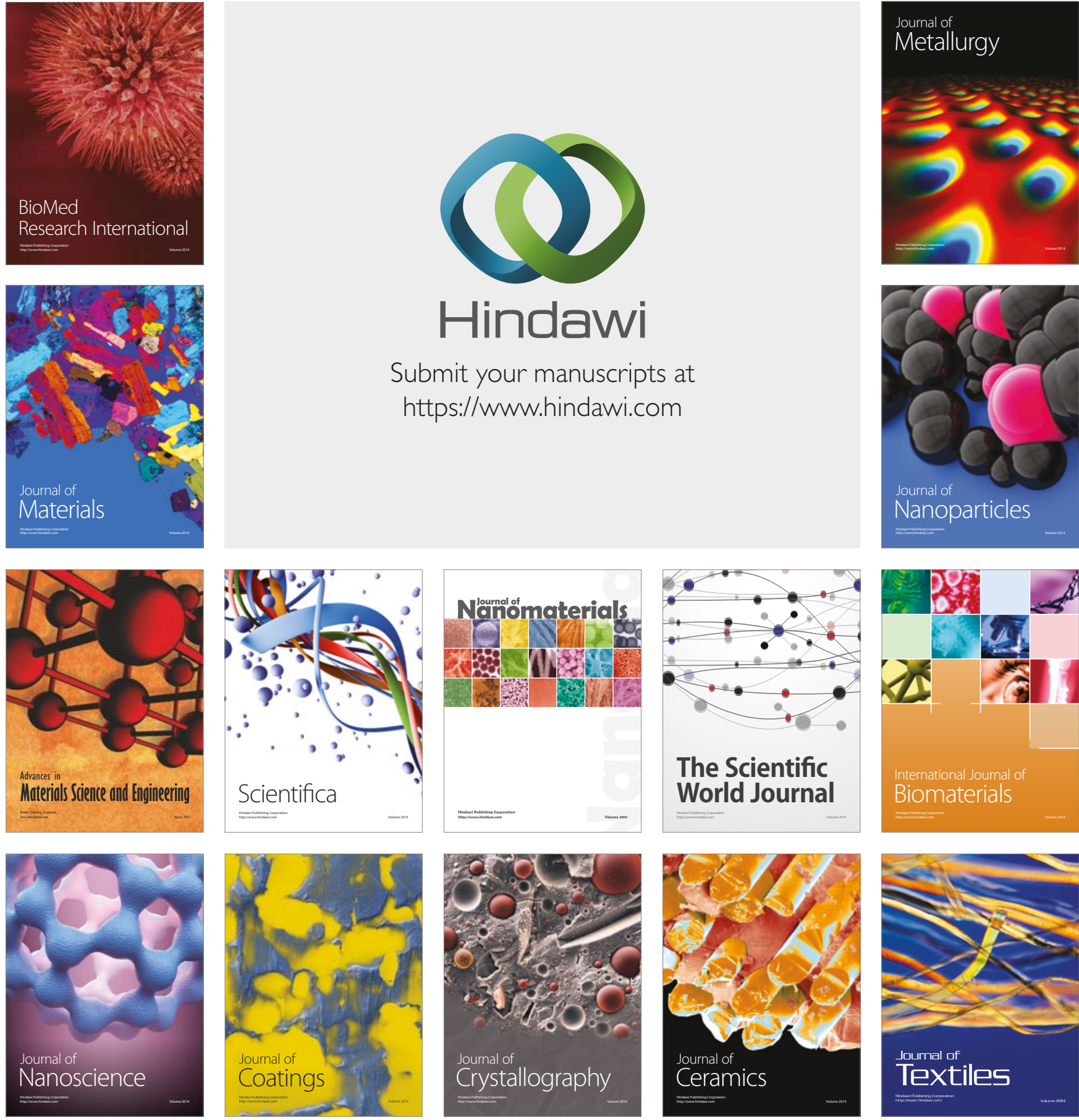

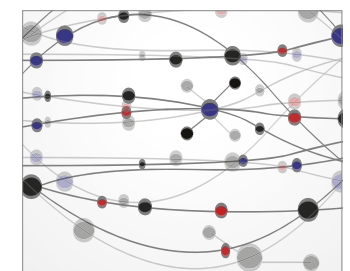

The Scientific World Journal
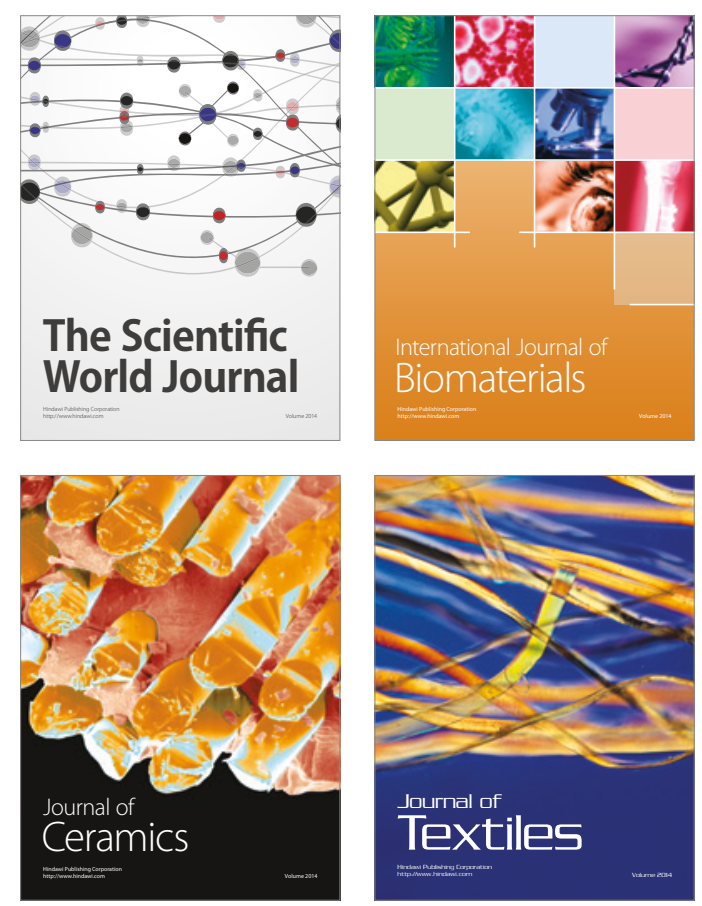